\title{
Comparing the disk-diffusion and agar dilution tests for Neisseria gonorrhoeae antimicrobial susceptibility testing
}

\author{
Hsi Liư ${ }^{*}$, Thomas H. Taylor Jr², Kevin Pettus ${ }^{1}$, Steve Johnson', John R. Papp ${ }^{1}$ and David Trees ${ }^{1}$
}

\begin{abstract}
Background: We assessed the validity of testing for antimicrobial susceptibility of clinical and mutant Neisseria gonorrhoeae (GC) isolates by disk diffusion in comparison to agar dilution, and Etest ${ }^{\circledast}$ (bioMerieux, France), respectively, for three third generation extended spectrum cephalosporins (ESC): ceftriaxone (CRO), cefixime (CFX), and cefpodoxime (CPD).

Methods: One hundred and five clinical isolates and ten laboratory-mutants were tested following Clinical Laboratory Standard Institute (CLSI) and manufacturer's standards for each of the three methods. The measured diameters by the disk diffusion method were tested for correlation with the MIC values by agar dilution. In addition, comparisons with the Etest ${ }^{\circledR}$ were made. Categorical results for concordance, based on standard CLSI cutoffs, between the disk diffusion and the other two methods, respectively, were tested using the Chi-square statistics. Reproducibility was tested for CFX across a 6-month interval by repeated disk tests.

Results: Across all 115 specimens, the disk diffusion tests produced good categorical agreements, exhibiting concordance of $93.1 \%, 92.1 \%$, and $90.4 \%$ with agar dilution and $93.0 \%, 92.1 \%$, and $90.4 \%$ with Etest ${ }^{\oplus}$, for CRO, CFX, and CPD, respectively. Pearson correlations between disk-diffusion diameters and agar dilution MIC's were $-0.59,-0$. 67, and -0.81 for CRO, CFX, and CPD, respectively. The correlations between disk diffusion and Etest ${ }^{\oplus}$ were $-0.58,-0$. 73, and -0.49. Pearson correlation between the CFX disk readings over a 6-month interval was $91 \%$.
\end{abstract}

Conclusions: Disk diffusion tests remain to be a useful, reliable and fast screening method for qualitative antimicrobial susceptibility testing for ceftriaxone, cefixime, and cefpodoxime.

Keywords: Antibiotic, Susceptibility testing, Ceftriaxone, Cefixime, Neisseria, Gonorrhoeae

\section{Background}

Neisseria gonorrhoeae (N. gonorrhoeae) is one of the most common sexually transmitted pathogens. Its high morbidity and associated medical and socio-economic costs make it one of the major public health issues in the U.S. and in the world. Approximately 300,000 cases are reported to the CDC each year [1]. Because many cases could be asymptomatic, a recent estimate indicates that there may be more than 820,000 infected individuals in the U.S [2]. Globally, many areas are experiencing significant rise in reported cases of gonorrhea. According to the World Health Organization (WHO), in

\footnotetext{
* Correspondence: hcl6@cdc.gov

${ }^{1}$ Division of STD Prevention, NCHHSTP, Atlanta, USA

Full list of author information is available at the end of the article
}

2008 there were 106 million new cases of gonorrhea worldwide [3].

CDC currently recommends that for uncomplicated genital, rectal, and pharyngeal gonorrhea, a combination of two drugs should be used; specifically, ceftriaxone $250 \mathrm{mg}$ is administered intramuscularly (IM) in a single dose, plus azithromycin $1 \mathrm{~g}$ orally in a single dose. When ceftriaxone is not available, cefixime $400 \mathrm{mg}$ orally in a single dose is recommended to replace ceftriaxone in combination with $1 \mathrm{~g}$ oral azithromycin $[4,5]$. Treatment guidelines also recommend individuals with pharyngeal infections return for medical consultation 1 week post therapy to ensure success of therapy.

It is not clear whether combining two antimicrobials with different mechanisms of action (e.g., ceftriaxone 
plus azithromycin) can delay the rise of isolates that are resistant to the extended spectrum cephalosporins (ESC) [6]. A recent report from Canada showed a significant decline in decreased susceptibility to cephalosporins but at the same time increased azithromycin resistance [7].

Despite the use of dual drug therapy, both CDC and the WHO have warned that gonorrhea will soon become more difficult to treat. This is because gonococcus is well known for its ability to develop antimicrobial resistance against first-line therapies within a short duration of a drug's introduction [8-11].

Until new drugs for treating gonorrhea are discovered, there is urgent need for public health professionals to closely monitor antimicrobial susceptibility of gonococci. Quick identification of isolates that potentially possess reduced susceptibility to cephalosporins is critical to controlling the spread of drug-resistant gonococcal organisms. The recent emergence of isolates with reduced antibiotic susceptibility across the country has further alerted physicians and epidemiologists to the urgent need to closely monitor such activities [10].

Three common tests are routinely used by laboratories to determine the antimicrobial susceptibility of $N$. gonorrhoeae. The agar dilution test is the gold standard and is used mostly by reference laboratories. We reported recently that the Etest ${ }^{\odot}$ (bioMerieux, France) is suitable to serve as an alternative test [12]. Both agar dilution and Etest methods report an MIC value which can be easily used by physicians or epidemiologists to determine treatment options. These two tests are appropriate for finding organisms with increased MIC values. While these tests are our first choice, they are not routinely used by all laboratories.

A third test, the disk diffusion test, is more commonly used by microbiological laboratories and hospitals world-wide to determine the antimicrobial susceptibility of many organisms against widespectrum antibiotics [9, 12-15]. The test is simple and widely accepted by many laboratories. Its use, however, is not without limitations. Disk diffusion test results are observed as a diameter of inhibition-zone. The diameter is relatively imprecise and often cannot convert to a MIC value, but rather provides a categorical classification of susceptible, intermediate, or resistant phenotypes [10]. For the treatment of gonococci with cephalosporins, only susceptible and nonsusceptible categories are accepted for classifications $[13,14,16]$.

In the past 20 years, the number of laboratories using the disk diffusion method for $N$. gonorrhoeae antimicrobial susceptibility testing has declined. In $1989,86 \%$ of public health laboratories surveyed used the disk diffusion method [17]. In a study performed in New York in 2000 , approximately $37.4 \%$ of laboratories used disk diffusion [18]; in a 2012 study [19], 47\% of laboratories were utilizing disk diffusion tests for $N$. gonorrhoeae antimicrobial susceptibility. Thus the disk diffusion method remains the choice of many reference and regional laboratories and hospitals to survey gonococcal drug susceptibility. However, without sufficient clinically ESC resistant isolates, it is not clear whether the disk diffusion method can reliably detect future ESC resistance.

In this report, we compared the disk diffusion method with the current gold standard agar dilution and a potential alternative Etest method. We analyzed the suitability and reliability of disk diffusion to monitor susceptibility of $N$. gonorrhoeae isolates of the most commonly used cephalosporins (ceftriaxone and cefixime). In addition, we included 10 laboratory generated mutants with raised MIC level against these common ESCs to simulate non-susceptible isolates. Cefpodoxime was also included in this study because it was used in selecting laboratory generated mutants.

\section{Methods \\ N. gonorrhoeae isolates}

One hundred and five confirmed $N$. gonorrhoeae isolates from various locations in the US, including Gonococcal Isolate Surveillance Project (GISP) isolates and reference strains, were used. In addition, 10 specimens of laboratory-derived mutants that are non-susceptible to cefpodoxime were selected. All 115 isolates were used in all three tests. All isolates were confirmed by passage and selection using the modified Thayer-Martin Medium (Scientific Resource Program [SRP], CDC).

\section{Mutation generation}

The laboratory generated mutations were selected for increased cephalosporin MICs by exposing parent strains SPN284 or GC3502 to elevated concentrations of cefpodoxime $(3.0 \mathrm{ug} / \mathrm{ml}$ or $4.5 \mathrm{ug} / \mathrm{ml})$ [20]. The specific concentrations of cefpodoxime were included in enriched GC agar base medium plates and 2$4 \times 10^{11}$ CFU of parent strain were inoculated. The resulting colonies were subcultured and maintained on GC agar base medium supplemented with $1 \%$ IsoVitaleX TM [Sigma-Aldrich, MO].

\section{Antimicrobial susceptibility testing}

Agar dilution tests and disk diffusion tests (BD BBL Sensi-Disc, Becton, Dickinson and Company, MD, U.S.A.) were performed by using the GC agar base medium supplemented with $1 \%$ IsoVitaleX TM. Both tests were performed according to the Clinical and Laboratory Standards Institute (CLSI) agar dilution or disk diffusion methods [21, 22]. The Etests were performed according to the manufacturer's recommendations (bioMerieux, France). Disk diffusion tests and Etests were 
performed at the same time while agar dilution tests were performed by itself due to the complexity nature of the test. Tests were prepared by suspending colonies of $N$. gonorrhoeae from an overnight culture of Chocolate II agar (Scientific Resources Program, SRP, CDC) into Muller Hinton Broth (Difco Laboratories, MI) and adjusted to an optical density (BioMate3, Thermo-Fisher Scientific) equal to that of a 0.5 McFarland standard. The organisms were evenly spread on the surface of a 10x150 mm GC base agar plate using a cotton swab and allowed to dry for about $10 \mathrm{~min}$ before the disks were applied to the plate and duplicated plates were performed. For the agar dilution method, a dilution of the suspension approximately $10^{4} \mathrm{CFU}$ per spot was inoculated within $15 \mathrm{~min}$ of preparation onto the GC base agar surface with a Steers inoculator. The plates were incubated at $35{ }^{\circ} \mathrm{C}$ in $5 \% \mathrm{CO}_{2}$ for $20-24$ h. The minimum inhibitory concentrations (MICs) were interpreted by reading growth inhibition (agar dilution) or the diameter of the inhibition zone measured using a ruler (disk diffusion). When replicates have different values, we report the test value which has smaller diameter or larger MIC. A panel of 7 quality control organisms: $N$. gonorrhoreae ATCC 49226, F28, P681E, CDC10328, CDC10329, SPJ15, and SPL4 were included in each assay for validation [23]. The disk diffusion and Etest methods were performed simultaneously [12]. The Agar dilution was performed according to CLIA specified guidelines.

\section{Statistical analyses}

Results between the disk diffusion, the agar dilution, and Etest can be compared and quantified using the CLSI cutoffs and categorical levels specified. Both agar dilution and Etest report MICs $(\mu \mathrm{g} / \mathrm{ml})$ which represent a continuous metric of per-unit concentrations. Disk diffusion provides an inhibition zone diameter which is also a continuous metric but in $\mathrm{mm}$ of distance. Accordingly, statistical comparisons between the MIC concentrations and the continuous metric diameter were performed using simple correlation and linear regression (Statistical Analysis System, Version 9.4). For all three tests, there exist CLSI standard cutoff values [18] to define susceptibility and non-susceptibility of the ESC spectrum antibiotics based on whether a particular test result is above, equal to, or below the cutoff. Hence the tests can be compared as a categorical outcome: susceptible (S) or non- susceptible (nS). Simple $2 \times 2$ tables and proportions concordant or not, between the pairwise test comparisons, are provided (Table 1). The specific threshold (cutoff) values for each test and drug are included in the table. To test for reproducibility, the disk diffusion test of cefixime was repeated at multiple time points over a 6-month period.

\section{Results}

The MICs measured by the agar dilution test and the diameter ( $\mathrm{mm}$, inhibition zone) of the disk diffusion test exhibit strong linear relationships for all three antibiotics. The Pearson's correlations between disk diffusion and agar dilution test were -0.59 , -0.67 , and $-0.81(p<0.0001)$, respectively, for ceftriaxone, cefixime, and cefpodoxime (Fig. 1a, b, c). Previously, the Etest was shown to perform comparably to the agar dilution test [12]. When disk diffusion was compared with Etest method, similar results were obtained. The correlations between disk diffusion and Etest for the three cephalosporins were $-0.58,-0.73$, and -0.49 , respectively. These results include all 115 pairs of tests, including clinical and mutant specimens, to insure no artificial range

Table 1 Categorical Comparisons of the Susceptibility Testing Results of Disk Diffusion with Agar Dilution

\begin{tabular}{|c|c|c|c|c|}
\hline \multicolumn{2}{|c|}{ Disk diffusion, zone diameter } & \multicolumn{3}{|c|}{ Agar dilution, MIC } \\
\hline & & Susceptible & Non-Susceptible & \\
\hline \multicolumn{2}{|l|}{ Ceftriaxone (CRO) } & $<=0.25 \mathrm{ug} / \mathrm{ml}$ & $>0.25 \mathrm{ug} / \mathrm{ml}$ & Totals \\
\hline Susceptible & $>=35 \mathrm{~mm}$ & 97 (84.4\%) & $0(0.00 \%)$ & 97 (84.4\%) \\
\hline \multirow[t]{2}{*}{ Non- Susceptible } & $<35 \mathrm{~mm}$ & $8(7.0 \%)$ & $10(8.7 \%)$ & $18(15.6 \%)$ \\
\hline & Totals & $105(91.3 \%)$ & $10(8.7 \%)$ & $115(100.0 \%)$ \\
\hline \multicolumn{2}{|l|}{ Cefixime (CFX) } & $<=0.25 \mathrm{ug} / \mathrm{ml}$ & $>0.25 \mathrm{ug} / \mathrm{ml}$ & Totals \\
\hline Susceptible & $>=31 \mathrm{~mm}$ & $94(81.7 \%)$ & $0(0.0 \%)$ & $94(81.7 \%)$ \\
\hline \multirow{2}{*}{ Non-Susceptible } & $<31 \mathrm{~mm}$ & $9(7.8 \%)$ & $12(10.4 \%)$ & $21(18.3 \%)$ \\
\hline & Totals & $103(89.6 \%)$ & $12(10.4 \%)$ & $115(100.0 \%)$ \\
\hline \multicolumn{2}{|l|}{ Cefpodoxime (CPD) } & $<=0.5 \mu \mathrm{g} / \mathrm{ml}$ & $>0.5 \mu \mathrm{g} / \mathrm{ml}$ & Totals \\
\hline Susceptible & $>=29 \mathrm{~mm}$ & $58(50.4 \%)$ & $7(6.1 \%)$ & $65(56.5 \%)$ \\
\hline \multirow[t]{2}{*}{ Non-Susceptible } & $<29 \mathrm{~mm}$ & $4(3.5 \%)$ & $46(40.0 \%)$ & $50(43.5 \%)$ \\
\hline & Totals & $62(53.9 \%)$ & $53(46.1 \%)$ & $115(100.0 \%)$ \\
\hline
\end{tabular}




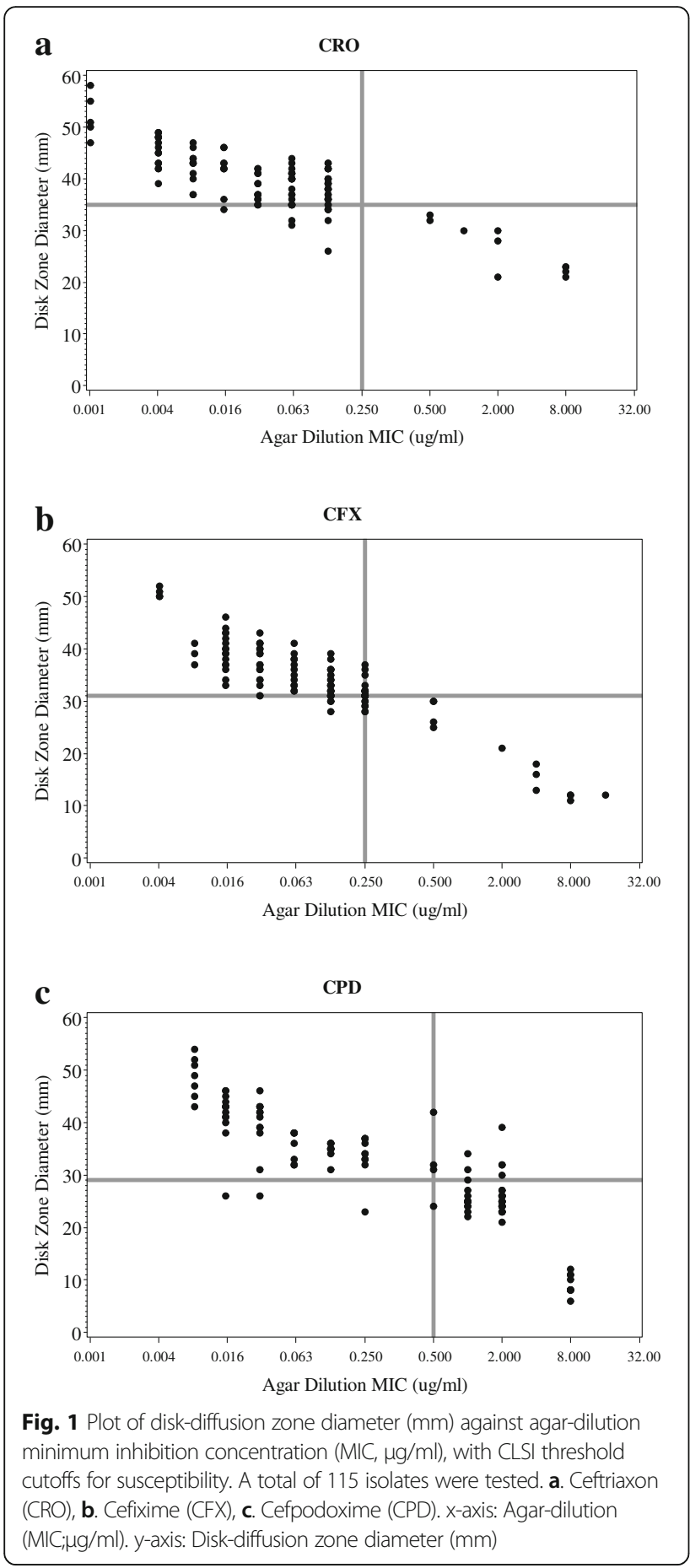

limitations on the correlations. The disk diffusion results agreed well with both tests across the full range of current clinical and potentially less susceptible strains for all three antibiotics.

The concordance of disk diffusion to agar dilution of the categorical results were $93.0 \%, 92.1 \%$, and $90.4 \%$ for ceftriaxone, cefixime, and cefpodoxime, respectively (Table 1). For disk diffusion and Etest, respective concordance rates were $93.0 \%, 92.1 \%$, and $90.4 \%$, respectively.

To test for reproducibility, the disk diffusion test of cefixime was repeated for each specimen. When the results of paired tests were compared, a 91\% correlation $(p<0.0001)$ was observed (Fig. 2).

\section{Discussion}

In this report, we studied the suitability and reliability of disk diffusion to monitor susceptibility of $N$. gonorrhoeae isolates of the most commonly used third generation cephalosporins (ceftriaxone and cefixime). The results and conclusions is limited to the three ESCs we tested and should not be extended without additional study to other molecules such as azithromycins or quinolones. We have demonstrated that the disk diffusion test had good correlations and categorical concordance when compared with current gold standard the agar dilution method (Fig. 1, Table 1). Disk diffusion also exhibited good reproducibility for cefixime (Fig. 2). This finding is important because the disk diffusion test remains a common method used by many regional laboratories and hospitals for detection of antimicrobial resistance, and the threat of reduced gonococcal susceptibility to extended spectrum cephalosporin may be imminent $[10$, 11, 24].

At present, there are few reported clinical cases of gonorrhea with isolates that demonstrate decreased ESC susceptibility [25-27]. In this study, we selected ten laboratory-generated mutants based on their significantly reduced in vitro susceptibility to three cephalosporins. These mutants also have non- susceptible phenotypes to cefixime and ceftriaxone as tested by agar dilution methods and Etest [12, 20] (Fig. 1). These highMIC mutants (MIC values: $2-8 \mathrm{ug}$ ) were used to simulate non-susceptible (or clinically resistant) phenotypes

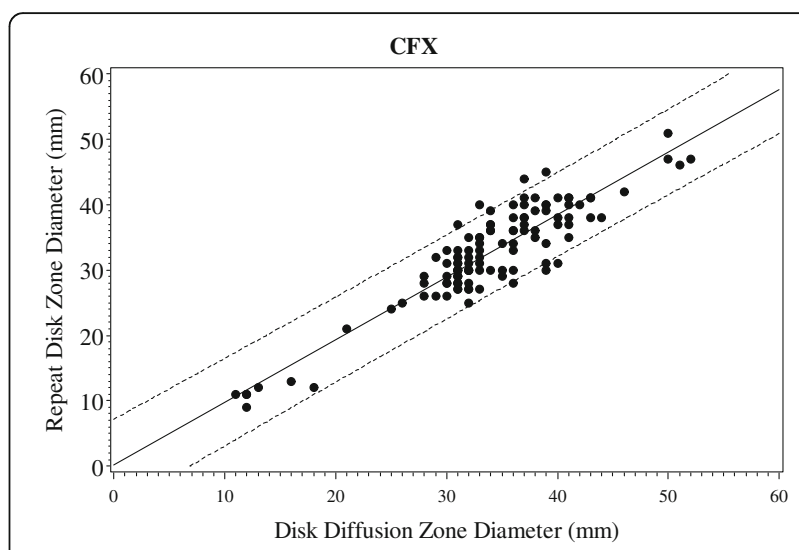

Fig. 2 Plot of disk-diffusion zone diameter $(\mathrm{mm})$ against itself at two different points in time, with fitted line and 95\% confidence band, for CFX. x-axis: Disk-diffusion zone diameter, time A (mm). y-axis: Repeat Disk-diffusion zone diameter, time B ( $\mathrm{mm}$ ) 
and test whether disk diffusion method could reliably detect future clinical isolates that are resistant to ESCs. All ten isolates showed good categorical agreement. This observation is important because it suggests that disk diffusion remains appropriate as a routine method to detect isolates with non-susceptibility phenotype.

GC-base medium and appropriate supplements (such as IsoVitaleX) are recommended by the CLSI for testing antibiotic susceptibility of $N$. gonorrhoeae species [16, $22]$. However, in some regions clinical laboratories routinely use Chocolate agar (e.g., Choc II) as testing medium. It has been reported that testing using the Chocolate agar generates results similar to those of GC base. However, a small discrepancy may result in missing isolates that fall into the potential non-susceptible category or generate false positives. Liao et al. have observed a $5.5 \%$ false resistance rate when chocolate agar is used compared with GC-base [28]. Thus, it is advisable that all laboratories using GC-base medium when performing tests of gonococcus.

According to the CLSI, the ESC antimicrobial susceptibility testing results for $N$. gonorrhoeae are classified into two categories, susceptible and non-susceptible; intermediate and resistant categories are not designated at this time $[21,22]$. For cefixime and ceftriaxone, isolates with MICs greater than $0.25 \mathrm{ug} / \mathrm{ml}$ (or $>=0.5 \mathrm{ug}$ / $\mathrm{ml}$ ) are considered not susceptible to these drugs. For epidemiological purposes the CDC sometimes classifies isolates with cefixime MICs $>=0.25 \mathrm{ug} / \mathrm{ml}$ and ceftriaxone MICs $>=0.125 \mathrm{ug} / \mathrm{ml}$ as isolates having reducedsusceptibility and an increase in numbers of reduced susceptibility is a warning sign for clinicians $[4,5,8]$. This type of assessment cannot be achieved using the disk diffusion test because at this time breakpoints (in $\mathrm{mm}$ ) are not available. Regardless, the GC-disk diffusion test appears appropriate for qualitative antibiotic susceptibility testing for clinicians to determine and choose the appropriate ESCs for treatment when no other testing alternatives are available.

However, the disk diffusion test has additional limitations that should be considered. Training and experience are required both for performing and reading the results. Further, the test is labor-intensive and has similar limitations to other culture-based tests. Reading the disk diffusion test can be subjective, human errors can affect the outcome, and despite good categorical classifications, it may have somewhat larger reproducibility variations than the Etest or agar dilution test. In this study, the same batch of disks was used throughout the experiments and the Etest strips were from one lot. Although different batches of agar plates were used, there is no reason to suspect that this variation had a major impact on results. In addition, one technician performed and read all tests. Given the nature of reading zone diameters and Etest strips, we might expect higher variability if results were read by multiple technicians.

\section{Conclusion}

For public health laboratories performing susceptibility testing of $N$. gonorrhoeae for ceftriaxone, cefixime, and cefpodoxime, disk diffusion test remains a viable method which produces results comparable to the current gold standard, agar dilution.

\begin{abstract}
Abbreviations
CFX: Cefixime; CLSI: Clinical Laboratory Standard Institute; CPD: Cefpodoxime; CRO: Ceftriaxone; ESC: Extended spectrum cephalosporins; GC: Neisseria gonorrhoeae; GISP: Gonococcal Isolate Surveillance Project; IM: Intramuscularly; MIC: Minimum inhibitory concentration; NS: Non-susceptible; S: Susceptible; SRP: Scientific Resource Program; WHO: World Health Organization;

Funding

Not applicable.

Availability of data and materials

Data will be shared with a reason.

\section{Authors' contributions}

All authors contributed equally and have approved the manuscript. The findings and conclusions in this report are those of the authors and do not necessarily represent the views of the Centers for Disease Control and Prevention. None of the authors have any competing interests with regard opinions or conclusions expressed in this manuscript.
\end{abstract}

\section{Competing interests}

The authors declare that they have no competing interests.

\section{Consent for publication}

Not applicable.

\section{Ethic approval and consent to participate}

Not applicable.

\section{Author details}

'Division of STD Prevention, NCHHSTP, Atlanta, USA. ${ }^{2}$ Division of Laboratory Systems, CSELS, Centers for Disease Control and Prevention, 1600 Clifton Rd, Atlanta, GA 30333, USA.

Received: 29 April 2016 Accepted: 3 November 2016

Published online: 24 November 2016

\section{References}

1. Centers for Disease Control and Prevention. 2014. Gonorrhea statistics. (http://www.cdc.gov/std/gonorrhea/stats.htm). Accessed 2 Apr 2105.

2. Satterwhite $\mathrm{CL}$, Torrone E, Meites E, Dunne EF, Mahajan R, Ocfemia MC, Su J, Xu F, Weinstock H. Sexually transmitted infections among US women and men: prevalence and incidence estimates, 2008. Sex Transm Dis. 2013;40:187-93.

3. World Health Organization. 2012. Global incidence and prevalence of selected curable sexually transmitted infections-2008. http://apps.who.int/iris/bitstream/ 10665/75181/1/9789241503839 eng.pdf. Accessed 15 Apr 2015.

4. Centers for Disease Control and Prevention. Update to CDC's Sexually Transmitted Diseases Treatment Guidelines, 2010: Oral Cephalosporins No Longer a Recommended Treatment for Gonococcal Infections. MMWR. 2012;61:590-4. http://www.cdc.gov/mmwr/preview/mmwrhtml/mm6131a3. htm. Accessed 15 Apr 2016.

5. Centers for Disease Control and Prevention. STD treatment guidelines, 2015. http// www.cdc.gov/mmwr/preview/mmwrhtm//rr6403a1.htm. Accessed 18 June 2015.

6. Rice LB. Will use of combination cephalosporin/azithromycin therapy forestall resistance to cephalosporins in Neisseria gonorrhoeae? Sex Transm Infect. 2015;91:238-40. doi:10.1136/sextrans-2014-051730.

7. Martin I, Sawatzky P, Liu G, Allen V, Lefebvre B, Hoang L, Drew S, Horsman G, Wylie J, Haldane D, Garceau R, Ratnam S, Wong T, Archibald C, Mulvey 
MR. Decline in decreased cephalosporin susceptibility and increase in azithromycin resistance in Neisseria gonorrhoeae. Canada Emerg Infect Disease. 2016;22:65-7. doi:10.3201/eid2201.151247.

8. Centers for Disease Control and Prevention. Cephalosporin susceptibility among Neisseria gonorrhoeae Isolates - United States, 2000-2010. MMWR Morb Mortal Wkly Rep. 2011;60:873-7.

9. Bala M, Singh V, Bhargava A, Ramesh V. Trends of resistance to antimicrobials recommended currently and in the past for management of gonorrhea in the Apex STD center in India and comparison of antimicrobial resistance profile between 2002-2006 and 2007-2012. Sex Transm Dis. 2015;42:218-22.

10. Bolan GA, Sparling PF, Wasserheit JN. The emerging threat of untreatable gonococcal infection. New Engl J Med. 2012;366:485-7.

11. Barry PM, Klausner JD. The use of cephalosporins for gonorrhea: the impending problem of resistance. Expert Opin Pharmacother. 2009;10:1-23.

12. Liu H, Taylor TH, Pettus K, Trees D. Assessment of Etest as an alternative to agar dilution for antimicrobial susceptibility testing of Neisseria gonorrhoeae. J Clin Microbiol. 2014;52:1435-40.

13. Clinical and Laboratory Standards Institute. Performance standards for antimicrobial disk susceptibility tests. Approved standard M2-A10. Wayne, PA: Clinical and Laboratory Standards Institute; 2009.

14. Bauer AW, Kirby WMM, Sherris JC, Turk M. Antibiotic susceptibility testing by a standardized single disk method. Am J Clin Pathol. 1996;45:493-6.

15. Jorgensen $\mathrm{JH}$, Ferraro MJ. Antimicrobial susceptibility testing: A review of general principles and contemporary practices. Clin Infect Dis. 2009;49: 1749-55.

16. Clinical and Laboratory Standards Institute. Performance standards for antimicrobial susceptibility testing: twenty-third informational supplement. CLSI document, Approved Standard M100-S24. Wayne, PA: Clinical and Laboratory Standards Institute; 2014.

17. Jones RN, Gavan TL, Thornsberry C, Fuchs PC, Gerlach EH, Knapp JS, Murray P, Washington II JA. Standardization of disk diffusion and agar dilution susceptibility tests for Neisseria gonorrhoeae: interpretive criteria and quality control guidelines for ceftriaxone, penicillin, spectinomycin, and tetracycline. J Clin Microbiol. 1989;27:2758-66.

18. Kiehlbauch JA, Hannett GE, Salfinger M, Archinal W, Monserrat C, Carlyn C. Use of the National Committee for Clinical laboratory Standards guidelines for disk diffusion susceptibility testing in New York state laboratories. J Clin Microbiol. 2000:38:3341-8.

19. Van T, Zanto S. 2012. STD testing practices in United States public health laboratories. National STD Prevention Conference. https://cdc.confex.com/ cdc/std2012/webprogram/Paper29915.html. Accessed 15 Apr 2015.

20. Johnson SR, Grad Y, Ganakammal SR, Burroughs M, Frace M, Lipsitch M, Wail $R$, Trees D. In vitro selection of Neisseria gonorrhoeae mutants with elevated MIC values and increased resistance to cephalosporins. Antimicrob Agents Chemother. 2014;58:6986-9.

21. Clinical and Laboratory Standards Institute. Development of in vitro susceptibility testing criteria and quality control parameters: approved guideline-3rd Edition. M23-A3E. Wayne, PA: Clinical and Laboratory Standards Institute; 2008

22. Clinical and Laboratory Standards Institute. Methods for antimicrobial susceptibility testing of anaerobic bacteria: approved standard-8th ed. CLSI document, Approved Standard M11-A8. Wayne, PA: Clinical and Laboratory Standards Institute; 2012

23. Centers for Disease Control and Prevention. February 2005. B-88: Neisseria gonorrhoeae reference strains for antimicrobial susceptibility testing. http:// www.cdc.gov/std/Gonorrhea/arg/B88-Feb-2005.pdf. Accessed 15 Apr 2016.

24. Jorgensen JH, Crawford SA, Fulcher LC, Glennen A, Harrington SM, Swenson J, Lynfield R, Murray PR, Tenover FC. Multi-laboratory evaluation of disk diffusion antimicrobial susceptibility testing of Neisseria meningitides isolates. J Clin Microbiol. 2006;44:1744-54.

25. Tapsall JW, Ndowa F, Lewis DA, Unemo M. Meeting the public health challenge of multidrug- and extensively drug-resistant Neisseria gonorrhoeae. Expert Rev Anti Infect Ther. 2009;7:821-34. http://dx.doi.org/10.1586/eri.09.63.

26. Unemo M, Golparian D, Syversen G, Vestrheim DF, Moi M. Two cases of verified clinical failures using internationally recommended firstline cefixime for gonorrhea treatment, Norway, 2010. Euro Surveill. 2010;15. http://www.eurosurveillance.org/ViewArticle.aspx?Articleld=19721. Accessed 15 Apr 2016.

27. Ohnishi M, Golparian D, Shimuta K, Saika T, Hoshina S, Iwasaku K, Nakayama S, Kitawaki J, Unemo M. Is Neisseria gonorrhoeae initiating a future era of untreatable gonorrhea? Detailed characterization of the first strain with high-level resistance to ceftriaxone. Antimicrob Agents Chemother. 2011;55: 3538-45.

28. Liao CH, Lai CC, Hsu MS, Chu FY, Wu MY, Huang YT, Hseuh PR. Antimicrobial susceptibility of Neisseria gonorrhoeae isolate determined by the agar dilution, disk diffusion and Etest methods: comparison of results using GC agar and chocolate agar. Int J Antimicrob Agents. 2010;35:457-60.

\section{Submit your next manuscript to BioMed Central and we will help you at every step:}

- We accept pre-submission inquiries

- Our selector tool helps you to find the most relevant journal

- We provide round the clock customer support

- Convenient online submission

- Thorough peer review

- Inclusion in PubMed and all major indexing services

- Maximum visibility for your research

Submit your manuscript at www.biomedcentral.com/submit
Biomed Central 